\title{
Symmetric dimethylation of poly-GR correlates with disease duration in C9orf72 FTLD and ALS and reduces poly-GR phase separation and toxicity
}

\author{
Lauren M. Gittings ${ }^{1,2,3}$. Steven Boeynaems ${ }^{4} \cdot$ Daniel Lightwood $^{5} \cdot$ Alison Clargo $^{5}$ - Sarfaraj Topia ${ }^{5}$ Lisa Nakayama ${ }^{4}$. \\ Claire Troakes ${ }^{6} \cdot$ David M. A. Mann $^{7} \cdot$ Aaron D. Gitler $^{4} \cdot$ Tammaryn Lashley $^{1,3} \cdot$ Adrian M. Isaacs $^{1,2} \mathbb{0}$
}

Received: 28 October 2019 / Revised: 22 November 2019 / Accepted: 22 November 2019 / Published online: 12 December 2019

(c) The Author(s) 2019

A GGGGCC repeat expansion in C9orf72 is the most common genetic cause of frontotemporal dementia (FTD) and amyotrophic lateral sclerosis (ALS). Pathologically, patients are characterised by TDP-43 pathology and distinct inclusions containing dipeptide repeat proteins (DPRs) that are produced by repeat associated non-ATG initiated translation of the repeat expansion. This produces five different DPRs: poly-GA, poly-GR poly-PR poly-AP and poly-GP. Poly-GR and poly-PR have been shown to be highly toxic in in vitro and in vivo models, but the mechanisms are not entirely clear [1]. We investigated whether methylation of arginine residues in poly-GR (which is much more abundant than poly-PR) contributes to disease pathogenesis. Three types of arginine methylation can occur, monomethylarginine

Electronic supplementary material The online version of this article (https://doi.org/10.1007/s00401-019-02104-x) contains supplementary material, which is available to authorized users.

\section{Adrian M. Isaacs}

a.isaacs@ucl.ac.uk

1 Department of Neurodegenerative Disease, UCL Queen Square Institute of Neurology, London WC1N 3BG, UK

2 UK Dementia Research Institute at UCL, Cruciform Building, Gower Street, London WC1E 6BT, UK

3 Queen Square Brain Bank for Neurological Disorders, UCL Queen Square Institute of Neurology, London, UK

4 Department of Genetics, Stanford University School of Medicine, Stanford, CA, USA

5 UCB Pharma, 216 Bath Road, Slough SL1 3WE, UK

6 London Neurodegenerative Diseases Brain Bank, Institute of Psychiatry, Psychology and Neuroscience, King's College London, London, UK

7 Division of Neuroscience and Experimental Psychology, School of Biological Sciences, University of Manchester, Salford Royal Hospital, Salford, UK
(MMA), or dimethylarginine in a symmetric (SDMA) or asymmetric (ADMA) confirmation. ADMA is the most prevalent modification with MMA and SDMA occurring at approximately 20-50\% that of ADMA [2]. The importance of arginine methylation in FTD and ALS has recently come to light as methylation of arginine residues within the FTD/ ALS-linked proteins FUS and hnRNPA2 is an important regulator of their liquid-liquid phase transition [7].

The presence of methylated DPRs has so far been suggested through indirect methods $[3,5,10]$. Therefore, in order to investigate DPR arginine methylation in C9orf72 FTD/ALS we generated and characterised two novel antibodies that detect the two forms of dimethylated poly-GR (Supplementary Fig. 1, online resource). In frontal cortex, they stained cytoplasmic inclusions only in $C 9$ orf 72 cases and did not label TDP-43 inclusions (Supplementary Figs. 2 and 3, online resource). A comparison to p62 inclusion numbers in the 15 C9orf 72 cases available at the Queen Square Brain Bank showed that $48 \%$ and $7 \%$ of p62 inclusions were positive for ADMA-GR and SDMA-GR respectively. These data show that arginine methylation of poly-GR is a common post-translational modification in $C 9$ orf 72 patient brain. Interestingly, a rare $C 9$ orf 72 homozygous case had few SDMA-GR inclusions (Supplementary Fig. 4, online resource), despite a high load of ADMA-GR, abundant DPR protein pathology and severe clinical features [6]. Given the early age of disease onset and short disease duration in the homozygous case, in conjunction with a low SDMA-GR burden, we hypothesised that SDMA-GR may correlate with clinical severity. To investigate this, the number of ADMAGR and SDMA-GR inclusions were quantified in the frontal cortex of 37 C9orf72 cases (C9orf72-FTLD $n=22$, C9orf72ALS $n=15$; details in Supplementary Table 1) and correlated with age at disease onset, age at death, disease duration and post-mortem delay (Supplementary Table 2). This identified a positive correlation between SDMA-GR and disease 
duration ( $r=0.5139, p=0.0026)$ (Fig. 1a), and age at death $(r=0.4568, p=0.0045)$ (Fig. 1b). The correlations remained significant when normalised to total DPR burden, measured by p62 staining, (Fig. 1c, d) (Supplementary Table 3), ruling out that SDMA-GR levels were simply reflecting total DPR levels. One possible interpretation of our finding that greater numbers of SDMA-GR are correlated with a longer disease duration and later age at death is that SDMA-GR is protective.

We therefore investigated the effect of methylation on GR phase separation and toxicity. We generated synthetic $(\mathrm{GR})_{20}$ peptides with or without ADMA/SDMA-modifications (Fig. 2a). Both ADMA- and SDMA-poly-GR displayed reduced phase separation, as evident from the higher saturation concentration assayed by turbidity measurements (Fig. 2b, c). Imaging showed that while droplets were less abundant for dimethylated poly-GR, they were larger in size (Fig. 2d). As we have previously reported for other basic peptides, such differences in droplet size stem from differences in the interaction strength of the phase separating
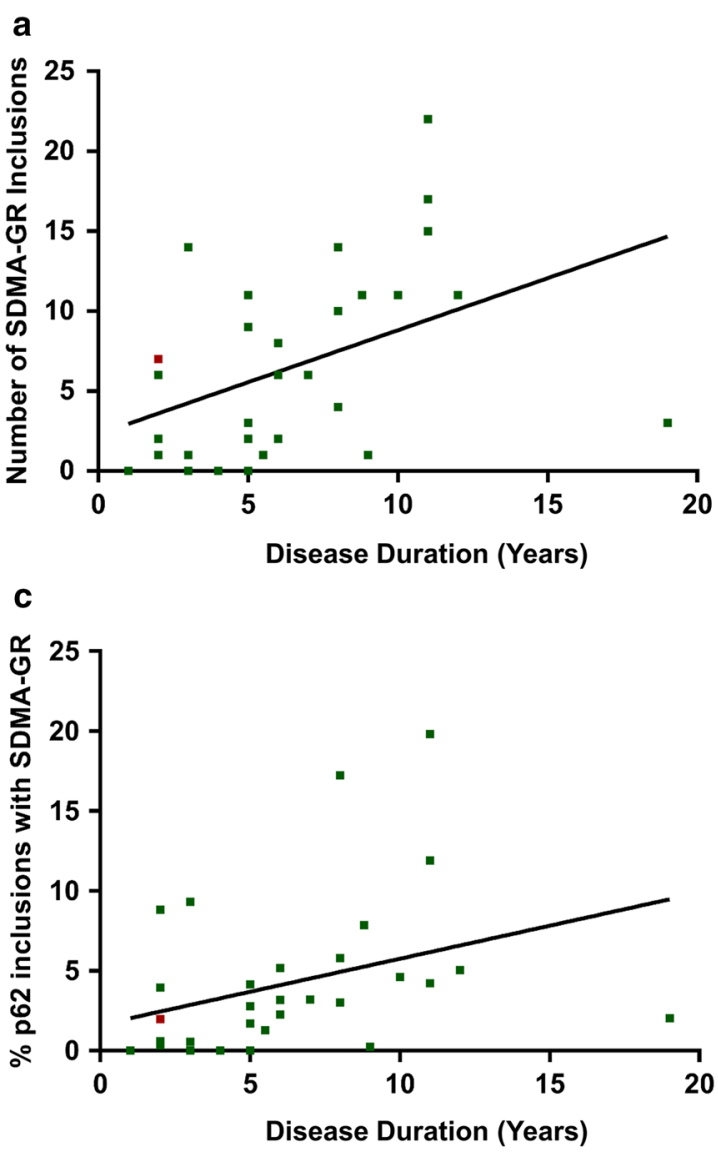

Fig. 1 SDMA-GR correlates positively with disease duration and age at death in $C 9$ orf 72 patients. Individual $C 9$ orf 72 cases are represented by green squares and the homozygous case as a red square. Positive correlation between the number of SDMA-GR inclusions and a disease duration $(r=0.51, p=0.0026, n=32)$, and $\mathbf{b}$ age at molecules [4]. Increased interaction strength increases droplet viscosity and surface tension, thereby reducing droplet fusion, leading to smaller droplets. Increased interaction strength will also lower the saturation concentration of phase separation. Hence, both turbidity measurements and droplet imaging point to a decrease in interaction strength between dimethylated poly-GR molecules. We next compared toxicity to primary neuronal cultures and found that dimethylated poly-GR was less toxic than unmethylated poly-GR (Fig. 2e-g), even though uptake of the peptides was similar (Supplementary Fig. 5, online resource). Hence, the same post-translational modifications that reduce poly-GR phase separation also reduces its neuronal toxicity.

A previous report found no correlation of poly-GR with neurodegeneration or clinical phenotypes [8], while two more recent studies showed poly-GR inclusions did correlate with neurodegeneration $[9,10]$. As these previous studies did not specifically label SDMA-GR, our findings provide new insights into the relationship of poly-GR inclusions with clinical phenotypes. Future studies are required

b

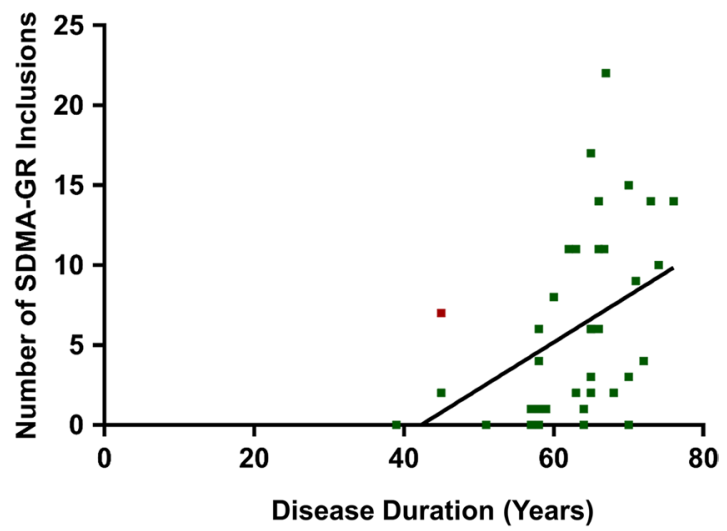

d

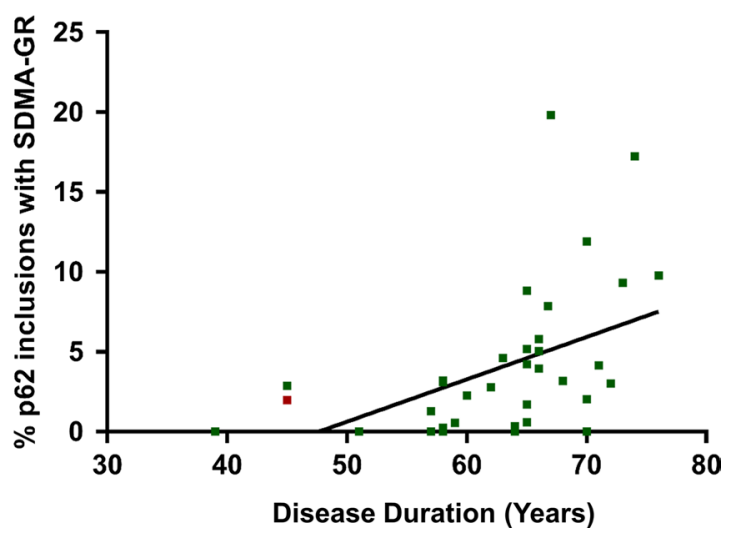

death $(r=0.46, p=0.0045, n=37)$. Positive correlation between the percentage of p62 inclusions with SDMA-GR and $\mathbf{c}$ disease duration $(r=0.47, p=0.0061, n=32)$ and $\mathbf{d}$ age at death $(r=0.55, p=0.0005$, $n=37)$. Spearman's rank correlation coefficient performed for all analyses 
a

GRGRGRGRGRGRGRGR... vs $\mathrm{G}^{*} \mathrm{GRG}{ }^{*} \mathrm{GRG}^{*} \mathrm{GRG}{ }^{*} \mathrm{GR} .$. $*$ SDMA/ADMA

b

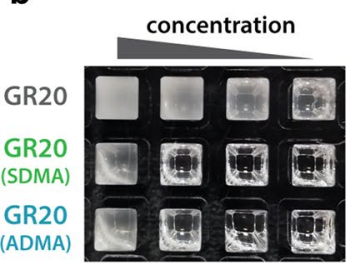

e

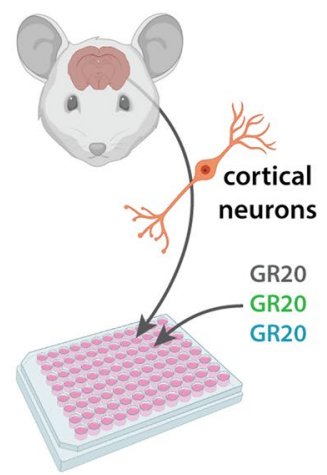

C

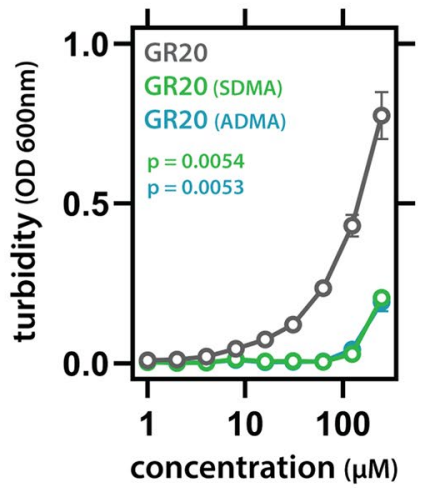

f

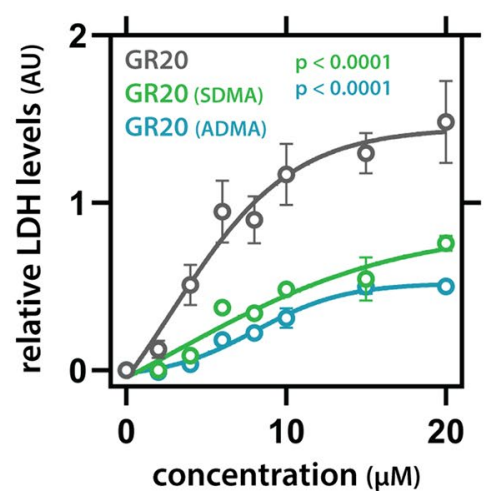

d

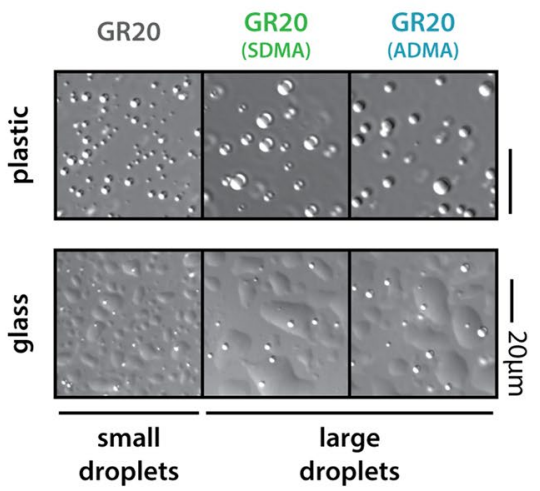

g

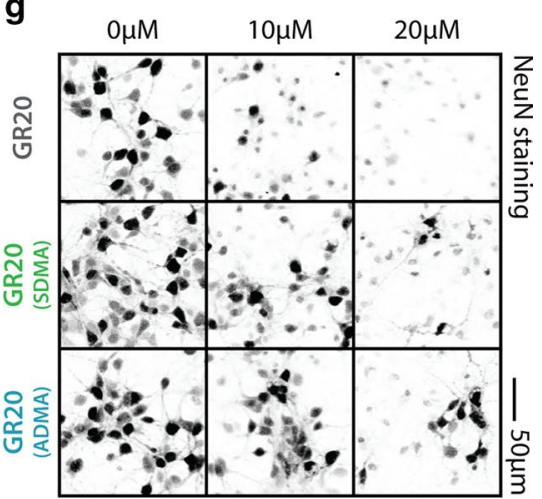

Fig. 2 Arginine dimethylation reduces phase separation and neurotoxicity of poly-GR. a Scheme showing methylation of synthetic $(\mathrm{GR})_{20}$ peptides. b Phase separation of $(\mathrm{GR})_{20}$ is reduced by SDMA and ADMA modification, as seen by a reduction in the concentrationdependent turbidity increase, quantified in $(\mathbf{c})$. Mean $(n=3)$ and SEM are shown. Two-way ANOVA. d Pictures showing increased droplet

to investigate why only SDMA-GR is associated with longer disease duration and age at death, and not ADMA-GR, as both forms were able to affect phase separation and toxicity (see Supplementary discussion, online resource for further discussion). In summary, our data show that arginine methylation is a common post-translation modification of poly-GR in $C 9$ orf72 patient brain that may influence disease course.

Open Access This article is licensed under a Creative Commons Attribution 4.0 International License, which permits use, sharing, adaptation, distribution and reproduction in any medium or format, as long as you give appropriate credit to the original author(s) and the source, provide a link to the Creative Commons licence, and indicate if changes were made. The images or other third party material in this article are included in the article's Creative Commons licence, unless indicated otherwise in a credit line to the material. If material is not included in the article's Creative Commons licence and your intended use is not permitted by statutory regulation or exceeds the permitted use, you will need to obtain permission directly from the copyright holder. To view a copy of this licence, visit http://creativecommons.org/licenses/by/4.0/. size of dimethylated $(\mathrm{GR})_{20}$. Concentration $250 \mu \mathrm{M}$. e Scheme of the neurotoxicity assay setup. $\mathbf{f}$ Quantification of exogenously added $(\mathrm{GR})_{20}$ toxicity to mouse primary cortical neurons. Mean $(n=4)$ and SEM are shown. Two-way ANOVA. g Pictures showing loss of neurons in $(\mathrm{GR})_{20}$-treated cultures (NeuN staining)

\section{References}

1. Balendra R, Isaacs AM (2018) C9orf72-mediated ALS and FTD: multiple pathways to disease. Nat Rev Neurol 14:544-558. https ://doi.org/10.1038/s41582-018-0047-2

2. Bedford MT, Clarke SG (2009) Protein arginine methylation in mammals: who, what, and why. Mol Cell 33:1-13. https://doi. org/10.1016/j.molcel.2008.12.013

3. Boeynaems S, Bogaert E, Michiels E, Gijselinck I, Sieben A, Jovicic A et al (2016) Drosophila screen connects nuclear transport genes to DPR pathology in c9ALS/FTD. Sci Rep 6:20877. https://doi.org/10.1038/srep20877

4. Boeynaems S, Holehouse AS, Weinhardt V, Kovacs D, Van Lindt J, Larabell C et al (2019) Spontaneous driving forces give rise to protein-RNA condensates with coexisting phases and complex material properties. Proc Natl Acad Sci USA 116:7889-7898. https://doi.org/10.1073/pnas.1821038116

5. Chitiprolu M, Jagow C, Tremblay V, Bondy-Chorney E, Paris G, Savard A et al (2018) A complex of C9ORF72 and p62 uses arginine methylation to eliminate stress granules by autophagy. Nat Commun 9:2794. https://doi.org/10.1038/s41467-01805273-7

6. Fratta P, Poulter M, Lashley T, Rohrer JD, Polke JM, Beck J et al (2013) Homozygosity for the C9orf72 GGGGCC repeat 
expansion in frontotemporal dementia. Acta Neuropathol 126:401-409. https://doi.org/10.1007/s00401-013-1147-0

7. Hofweber M, Dormann D (2019) Friend or foe-post-translational modifications as regulators of phase separation and RNP granule dynamics. J Biol Chem 294:7137-7150. https://doi. org/10.1074/jbc.TM118.001189

8. Mackenzie IR, Frick P, Grasser FA, Gendron TF, Petrucelli L, Cashman NR et al (2015) Quantitative analysis and clinicopathological correlations of different dipeptide repeat protein pathologies in C9ORF72 mutation carriers. Acta Neuropathol 130:845-861. https://doi.org/10.1007/s00401-015-1476-2

9. Saberi S, Stauffer JE, Jiang J, Garcia SD, Taylor AE, Schulte D et al (2018) Sense-encoded poly-GR dipeptide repeat proteins correlate to neurodegeneration and uniquely co-localize with TDP-43 in dendrites of repeat-expanded C9orf72 amyotrophic lateral sclerosis. Acta Neuropathol 135:459-474. https://doi.org/10.1007/ s00401-017-1793-8

10. Sakae N, Bieniek KF, Zhang YJ, Ross K, Gendron TF, Murray ME et al (2018) Poly-GR dipeptide repeat polymers correlate with neurodegeneration and clinicopathological subtypes in C9ORF72related brain disease. Acta Neuropathol Commun 6:63. https://doi. org/10.1186/s40478-018-0564-7

Publisher's Note Springer Nature remains neutral with regard to jurisdictional claims in published maps and institutional affiliations. 